\title{
ESTIMATIVA DO COEFICIENTE DE EXPANSÃO TÉRMICA DA GEMA DE OVO LÍQUIDA
}

\author{
César Augusto CANCIAM*
}

\author{
"Mestre em Engenharia Química, professor lotado no Departamento Acadêmico de Engenharia Química da \\ Universidade Tecnológica Federal do Paraná - Câmpus Ponta Grossa, canciam@utfpr.edu.br
}

Recebido em: 02/02/2015 - Aprovado em: 15/06/2015 - Disponibilizado em: 15/07/2015

\begin{abstract}
RESUMO:
$\mathrm{O}$ estudo da dilatação térmica em líquidos é feito somente em relação à dilatação volumétrica, sendo importante o conhecimento do coeficiente de expansão térmica. O objetivo deste trabalho foi estimar o coeficiente de expansão térmica da gema de ovo líquida a partir da análise de regressão linear de dados experimentais da massa específica em função da temperatura. Esses dados experimentais foram obtidos de outro trabalho. $\mathrm{O}$ valor encontrado foi de 5,0312 $\mathrm{x}$ $10^{-50} \mathrm{C}^{-1}$, em um intervalo de temperatura de $0,4 \mathrm{a} 60,8^{\circ} \mathrm{C}$. Na literatura, não consta valor experimental para o coeficiente de expansão térmica da gema de ovo líquida, tornando-se difícil a comparação com valor encontrado.
\end{abstract}

PALAVRAS-CHAVE: Dilatação. Gema de ovo líquida. Estimativa. Regressão linear.

\section{ESTIMATE OF THE THERMAL EXPANSION COEFFICIENT OF THE LIQUID EGG YOLK}

\begin{abstract}
:
The study of the thermal dilatation in liquids is only made in relation to the volumetric dilatation, but it is also important to know the thermal expansion coefficient. The objective of this work was to estimate the thermal expansion coefficient of the liquid egg yolk from the linear regression analysis of the experimental data specific weight in a function of temperature. These experimental data were obtained from other work. The value obtained was $5.0312 \times 10^{-50} \mathrm{C}^{-1}$, in a temperature range of 0.4 to $60.8^{\circ} \mathrm{C}$. In the literature, not reported experimental value for the thermal expansion coefficient of the liquid egg yolk, making it difficult to compare with value obtained.
\end{abstract}

KEYWORDS: Dilatation. Liquid egg yolk. Estimative. Linear Regression.

\section{INTRODUÇÃO}

As propriedades térmicas são resultados da padronização e dimensionamento dos cálculos de engenharia, e tais informações estão associadas com a resposta ou reação dos materiais à aplicação de calor. São consideradas como propriedades térmicas: a capacidade calorífica, a dilatação térmica e a condutividade térmica conforme descreve Incropera e Dewitt (1998) e Callister (2002).

Os materiais tendem a reagir diferentemente a uma variação da temperatura. Alguns materiais apresentam uma grande variação nas suas dimensões com o aumento da temperatura, enquanto outros praticamente não mudam suas dimensões. Em especial, tal comportamento é relacionado com a proximidade das moléculas, e 
consequentemente ao estado físico que o constituinte se apresenta (PADILHA, 1997).

Jerônimo, Balbino e Fernandes (2012) comentam que a dilatação térmica é variável e tende a manter uma relação direta com a composição química dos diferentes líquidos.

Os líquidos, ao contrário dos sólidos, apresentam somente volume definido; enquanto que os sólidos, forma própria e volume definido. Dessa forma, o estudo da dilatação térmica em líquidos é feito somente em relação à dilatação volumétrica, sendo por isso, importante o conhecimento do coeficiente de expansão térmica (CARRON e GUIMARÃES, 1997).

Segundo Canciam (2014), o conhecimento do coeficiente de expansão térmica de um material permite estudar o comportamento da dilatação volumétrica desse material, avaliar os impactos no sistema de medição volumétrico devido à variação da temperatura, servir de dado no projeto de equipamentos e acessórios, considerando possíveis dilatações resultantes de fortes mudanças de temperatura; corresponder a uma das variáveis na estimativa da tensão volumétrica e da entalpia de vaporização e estudar a convecção livre que influencia na transferência de calor em tubulações.

O coeficiente de expansão térmica $(\beta)$ indica a variação do volume $(V)$ provocada pela variação da temperatura $(T)$, enquanto a pressão $(P)$ permanece constante, sendo definido como (NETZ e ORTEGA, 2008):

$$
\beta=\left(\frac{1}{V}\right) \cdot\left(\frac{\partial V}{\partial T}\right)_{P}
$$

Em termos da massa específica, a Equação (1) pode ser reescrita na forma de (CANCIAM, 2012):

$$
\ln \left(\frac{\rho_{0}}{\rho}\right)=\beta \cdot\left(T-T_{0}\right)
$$

Em que $\rho_{0}$ corresponde à massa específica do material na temperatura inicial $T_{0}$ e $\rho$, à massa específica na temperatura final $T$.

A Equação (2) corresponde a uma função afim, ou seja, o gráfico de $\ln \left(\frac{\rho_{0}}{\rho}\right)$ versus $\left(T-T_{0}\right)$ fornece uma reta em que o coeficiente angular é numericamente igual ao coeficiente de expansão térmica $\beta$ (CANCIAM, 2010).

Jerônimo, Balbino e Fernandes (2012) comentam que o coeficiente de expansão térmica está associado à energia de ligação química entre as espécies (átomos ou moléculas). Materiais em que as ligações químicas são fortes apresentam baixos coeficientes de expansão térmica. Isto porque a dilatação térmica está associada à variação assimétrica da energia de ligação com a distância entre as espécies. Durante o aquecimento, as espécies aumentam a frequência e a amplitude de vibração. Como as forças de repulsão são sempre maiores que 
as forças de atração, a distância média entre as espécies também aumenta.

A gema de ovo é formada de uma mistura de proteínas complexas compostas, basicamente, por glicoproteínas, fosfoglicoproteínas, lipoproteínas e fosfoglicolipoproteínas (SGARBIERI, 1996). Por centrifugação, a gema do ovo é decomposta em duas fases, os sedimentos sólidos $(52 \%)$ e o sobrenadante constituído por água (48\%). Nos sólidos destacam duas frações: as frações de baixa densidade e as frações de alta densidade (OLIVEIRA et al., 2001).

A fração proteica de baixa densidade parece possuir uma estrutura em micelas, contendo fosfolipoproteínas em que a porção rica em lipídios neutros constitui a parte central da micela enquanto que a parte fosfolipídica e proteica posicionariam na superfície da micela. As forças que dão estabilidade a esse sistema formado por lipídios, fosfolipídios e glicoproteínas são do tipo interações hidrofóbicas ou de Van der Waals (interações fracas) (SGARBIERI, 1996).

A fração de elevada densidade é composta basicamente por lipovitelinas e fosvitina. Enquanto as primeiras são estabilizadas por interações hidrofóbicas ou de Van der Waals, a segunda comporta-se como polieletrólito; com a capacidade de ligar fortemente com vários íons e substâncias polares. Este tipo de interação é denominada de interação íon - molécula polar; é uma força forte e sua magnitude pode ser comparável à de uma ligação covalente (SGARBIERI, 1996).

Dessa forma, o objetivo deste trabalho foi estimar o coeficiente de expansão térmica da gema de ovo líquida a partir da análise de regressão linear de dados experimentais da massa específica em função da temperatura.

\section{MATERIAIS E MÉTODOS}

A Tabela 1 relaciona os dados experimentais da massa específica da gema de ovo líquida em função da temperatura.

Tabela 1. Efeito da temperatura sobre a massa específica da gema de ovo líquida

\begin{tabular}{cc}
\hline Temperatura $\left({ }^{\circ} \mathrm{C}\right)$ & $\begin{array}{c}\text { Massa específica } \\
\left(\mathrm{kg} \cdot \mathrm{m}^{-3}\right)\end{array}$ \\
\hline 0,4 & 1133,2 \\
8,9 & 1132,6 \\
20,3 & 1132,1 \\
28,4 & 1131,6 \\
37,8 & 1131,0 \\
48,2 & 1130,4 \\
55,2 & 1130,0 \\
60,8 & 1129,8 \\
\hline
\end{tabular}

Fonte: Adaptado de Gut et al (2005).

Para a obtenção do coeficiente de expansão térmica foi utilizada a metodologia proposta por Canciam (2005), que considera a análise de regressão linear de dados de $\ln \left(\frac{\rho_{0}}{\rho}\right)$ em função de $\left(T-T_{0}\right)$.

Com a aplicação dos dados indicados na Tabela 1 na Equação 2, foi possível estimar o coeficiente de expansão térmica a partir da análise de regressão linear, de 
maneira que, o coeficiente angular da reta obtida pelo gráfico de $\ln \left(\frac{\rho_{0}}{\rho}\right)$ versus $\left(T-T_{0}\right)$ corresponde numericamente ao valor do coeficiente de expansão térmica $(\beta)$.

A análise de regressão linear foi realizada utilizando um software específico disponível na calculadora CASIO FX-850P Scientific Library 116.

Nos cálculos, a temperatura inicial $T_{0}$ correspondeu a $0,4^{\circ} \mathrm{C}$ e a massa específica $\rho_{0}$, a $1133,2 \mathrm{~kg} \cdot \mathrm{m}^{-3}$.

\section{RESULTADOS E DISCUSSÃO}

A Figura 1 ilustra o gráfico de $\ln \left(\frac{\rho_{0}}{\rho}\right)$ versus $\left(T-T_{0}\right)$ para a gema de ovo líquida.

Figura 1: Gráfico de $\ln \left(\frac{\rho_{0}}{\rho}\right)$ versus $\left(T-T_{0}\right)$ para a gema de ovo líquida

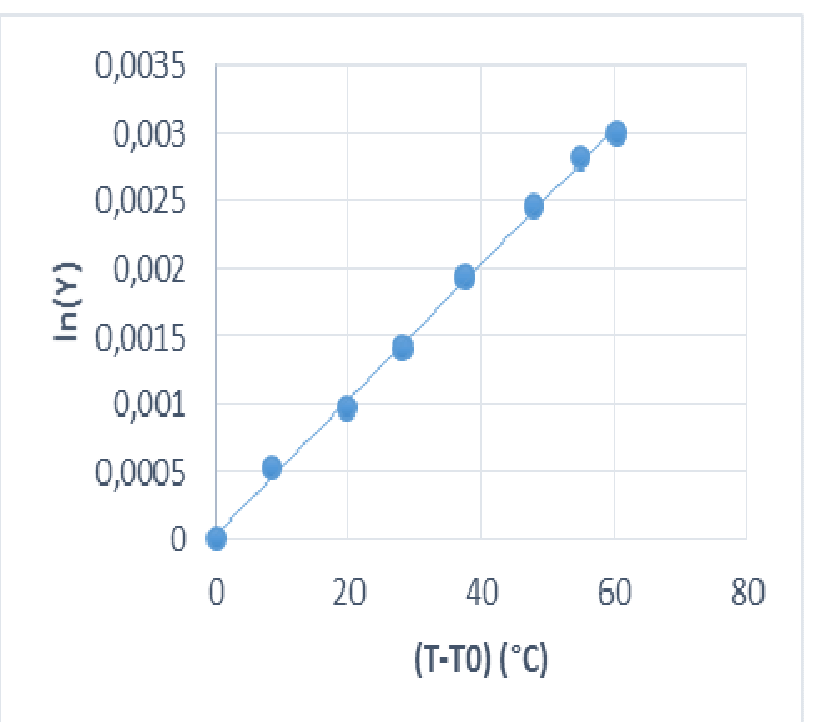

$\mathrm{Na}$ Figura 1, a razão $\left(\frac{\rho_{0}}{\rho}\right)$ é representada por $\mathrm{Y}$.

A análise de regressão linear forneceu como resultado para o coeficiente de expansão térmica da gema de ovo líquida o valor de $5,0312 \times 10^{-50} \mathrm{C}^{-1}$. Tendo como valor para o coeficiente de correlação $\left(R^{2}\right)$, o valor de 0,9989 .

Com relação ao coeficiente de correlação $\left(R^{2}\right)$, Pinheiros e coautores (2009) comentam que esse parâmetro mede a interdependência linear entre as variáveis e avalia a qualidade do ajuste, ou seja, quanto mais próximo o coeficiente de correlação for da unidade, melhor o ajuste da reta em relação aos pontos da dispersão.

Lira (2004) fornece uma classificação para as correlações lineares. A autora comenta que a correlação linear é classificada como muito forte quando os valores do coeficiente de correlação são maiores ou iguais a 0,90 e menores que 1,0. Dessa forma, observa-se que a correlação linear é classificada como muito forte para a gema de ovo líquida.

Foi realizada uma pesquisa na literatura a fim de obter valores do coeficiente de expansão térmica da gema de ovo líquida e constatou-se a ausência de valores experimentais para essa grandeza. Dessa forma, dificulta a análise dos valores obtidos neste trabalho.

Fonte: Autor. 
De acordo com Cabral e Lago (2002), o coeficiente de expansão térmica da água equivale a $2,07 \times 10^{-4 o} \mathrm{C}^{-1}$.

A molécula de água é uma molécula polar. A interação entre suas moléculas é do tipo ligação de hidrogênio. A ligação de hidrogênio ocorre quando um átomo de hidrogênio liga-se por covalência a um átomo mais eletronegativo e mantém uma afinidade residual por outro átomo eletronegativo, apresentando uma tendência à carga positiva (BROWN e HOLME, 2009).

Comparando os coeficientes de expansão térmica da água e da gema de ovo líquida, percebe-se que o coeficiente de expansão térmica da água é maior que o coeficiente de expansão térmica da gema de ovo liquida. Provavelmente devido as interações existentes entre a fosvitina e a água (interações do tipo íon - molécula polar) que ocorrem na gema de ovo líquida.

De acordo com Brown e Holme (2009), as interações do tipo íon - molécula polar são mais fortes em relação às ligações de hidrogênio. Isto justificaria o fato de que o coeficiente de expansão térmica da gema do ovo líquida é menor que o coeficiente de expansão térmica da água.

Todas essas considerações necessitam ser mais estudadas e discutidas.

Segundo Santos e Vieira (2010), a partir do conhecimento do coeficiente de expansão térmica é possível avaliar os impactos no sistema de medição volumétrico.
Neste sentido, considerando um volume de 1000 litros de água com uma variação de temperatura de $10^{\circ} \mathrm{C}$, o volume de água aumenta em torno de 2,07 litros. Com esse mesmo raciocínio, o volume de 1000 litros de gema de ovo líquida, com uma variação de temperatura de $10^{\circ} \mathrm{C}$, o volume da gema de ovo líquida aumenta em torno de 0,50 litro.

\section{CONCLUSÃO}

O objetivo deste trabalho foi estimar o coeficiente de expansão térmica da gema de ovo líquida. Para tanto, foi realizada uma análise de regressão linear de dados experimentais da massa específica da gema de ovo líquida em função da temperatura.

Para um intervalo de temperatura entre 0,4 e $60,8^{\circ} \mathrm{C}$, o valor encontrado para o coeficiente de expansão térmica da gema de ovo líquida foi igual a $5,0312 \times 10^{-5 o} \mathrm{C}^{-1}$.

O coeficiente de correlação encontrouse próximo da unidade, classificando a correlação linear como muito forte.

Em comparação com a água, constatou-se que a dilatação volumétrica da gema de ovo líquida é menor em relação a água, provavelmente devido às interações existentes entre a fosvitina e a água presentes na gema de ovo líquida. 


\section{REFERÊNCIAS}

BROWN, L.S.; HOLME, T.A. Química geral aplicada à Engenharia. Cengage Learning, São Paulo, 2009. 653p.

CABRAL, F.; LAGO, A. Física 2. Harbra, São Paulo, 2002. 516p.

CALLISTER, W.D. Ciência e Engenharia de Materiais: uma introdução. Livros Técnicos e Científicos Editora, Rio de Janeiro, 2002. 450p.

CANCIAM, C.A. Predição do coeficiente de expansão térmica do óleo de algodão. Publicatio UEPG - Ciências Exatas e da Terra, Agrárias e Engenharias, v. 11, n. 3, p.27-31, 2005.

CANCIAM, C.A. Predição do coeficiente de expansão térmica do óleo de gergelim (Sesamum indicum L.) através da aplicação da análise de regressão linear. E-xacta, v. 3, n. 1, p.21-28, 2010.

CANCIAM, C.A. Predição do coeficiente de expansão térmica do óleo de girassol através da aplicação da análise de regressão linear. Revista Brasileira de Tecnologia Agroindustrial, v. 6, n. 2, p.852-863, 2012.

CANCIAM, C.A. Estudo do coeficiente de expansão térmica de soluções aquosas de soda cáustica. Revista de Engenharia e Tecnologia, v. 6, n. 3, p.72-79, 2014.

CARRON, W.; GUIMARÃES, O. As Faces da Física. Moderna, São Paulo, 1997. 277p.

GUT, J.A.W.; PINTO, J.M.; GABAS, A.L.; TELIS-ROMERO, J. Continuous pasteurization of egg yolk: thermophysical properties and process simulation. Journal of Food Process Engineering, v. 28, n. 2, p. 181203, 2005.

INCROPERA， F.P.; DEWITT, D.P. Fundamentos de transferência de calor e massa. Livros Técnicos e Científicos Editora, Rio de Janeiro, 1998. 494p.

JERÔNIMO， C.E.M.; BALBINO， C.P.; FERNANDES, H.G. Coeficiente de dilatação volumétrica determinados pela curva ASTM em frações de petróleo. Scientia Plena, v.8, n. 9, p. 1-8, 2012.

LIRA, S. A. Análise de correlação: abordagem teórica e de construção dos coeficientes com aplicação. Dissertação de Mestrado do Programa de Mestrado em Ciências da Universidade Federal do Paraná, 2004. 196p.

NETZ, P.A.; ORTEGA, G.G. Fundamentos de físico-química: uma abordagem conceitual 
para as ciências farmacêuticas. Artmed, Porto Alegre, 2008. 299p.

OLIVEIRA， B.L.; VALLE， R.H.P.; BRESSAN, M.C.; CARVALHO, E.P. Tecnologia de ovos. UFLA/FAEPE Editora, Lavras, 2001. 75p.

PADILHA, A.F. Materiais de Engenharia: microestrutura e propriedades. Hemus, São Paulo, 1997. 349p.

PINHEIROS, J.I.D.; CUNHA, S.B.; CARVAJAL, S.R.; GOMES, G.C. Estatística básica: a arte de trabalhar com dados. Elsevier, Rio de Janeiro, 2009. 295p.

SANTOS, D.Q.; VIEIRA, D.F. Determinação de coeficiente de expansão térmica do biodiesel e seus impactos no sistema de medição volumétrico. Eclética Química, v. 35, n. 4, p. 107-112, 2010.

SGARBIERI, V.C. Proteínas em alimentos protéicos. Livraria Varela Editora, 1996. $517 \mathrm{p}$. 